\title{
MODERNIZATION OF CHINESE PHOTOELECTRIC ASTROLABES AND IMPROVEMENT OF OPTICAL REFERENCE SYSTEM
}

\author{
LI DONG-MING ${ }^{1}$, WANG HONG-QI ${ }^{2}$, ZHAO GANG ${ }^{3}$, WANG ZE-ZHI ${ }^{4}$, AND \\ WANG RUI ${ }^{5}$ \\ ${ }^{1}$ Purple Mountain Observatory, Nanjing, China \\ ${ }^{2}$ Shanxi Observatory, Xian, China \\ ${ }^{3}$ Shanghai Observatory, Shanghai, China \\ ${ }^{4}$ Beijing Observatory, Beijing, China \\ ${ }^{5}$ Yunnan Observatory, Kunming, China
}

The features of the astrolabe are that:

(1) a span of 6 hour of R.A. of star positions can be tied in a group of observation of 2 hours, allnight observation can cover more than half of the sky, and

(2) the declination system can be set up with high precision without the need of a precise circle $(\mathrm{Li}$ 1987).

Observation with the astrolabe will make an important contribution to the improvement of the fundamental reference system (Fricke 1972, 1985; Guinot et al. 1961; Li 1987).

In the past 3 years, we have reequipped the 4 photoelectric astrolabes (1 Mark I and 3 Mark II). The modernization of Chinese photoelectric astrolabes includes the following:

(a) Automation of observational process. Now these instruments can operate automatically all night and observe about 40 stars per hour.

(b) modernization of photoelectric record technique to extend the limiting magnitude to about 10th magnitude or fainter. The later part of the work will be finished early next year.

The Chinese photoelectric astrolabes will first contribute to the improvement and expansion of the FK5 and will obtain valuable data to the linking of radio/optical, space/ground-based reference frames around 1994. Careful comparison of modern astrolabe catalogs, meridian catalogs and space catalogs will be conducive to the research on the sources and magnitude of systematic errors of ground-based catalogs from various angles, will help to explore the possibility to improve the fundamental proper motion system in the light of the revealed systematic errors and will find out the trend of development of various techniques.

The working program to improve the fundamental reference system with Chinese photoelectric astrolabes is limited to the northern sky at present. We have noticed that South American colleagues are carrying out astrolabe observations of bright stars on the southern sky (Clauzet 1986). The 
importance of this work will be more remarkable if the observations can be extended to SRS stars (IAU Comm.8 1986). Chinese astronomers are interested in cooperating with foreign colleagues, especially those in South America, to accomplish this task.

While carrying out the observations mentioned above, we will pay particular attention to determination of precise positions of radio stars in the fundamental optical reference system. $86 \mathrm{high}$ priority Hipparcos radio stars with $\delta>-4^{\circ}$ will be included in the observation program. The observation will be extended to $\delta=-15^{\circ}$ with the astrograph. It is expected that precise positions of more than 110 radio stars will be obtained. Among them about 50 will be observed with both astrolabe and astrograph. The astrolabe has strong capability of smoothing local errors. Optical positions of radio stars determined with the astrolabe refer to a fundamental position system of large range. Comparison of the results of astrolabe, meridian and photography will be conducive to revealing possible systematic differences between defferent techniques and the causes of systematic difference in radio-FK5.

Besides determination of star positions, the modernized photoelectric astrolabe can obtain photometric data and resolve binaries to a certain extent. It can be used to observe planets and astroids. At present, 4 Chinese photoelectric astrolabes are engaged in observation of 2000 bright stars. After it is accomplished in early next year, they will be put into the observation program mentioned above, including some stars of astrophysical interest.

\section{References}

Clauzet, L.B.F.: 1986, Transactions of the IAU, XIXB, 121.

Fricke, W.: 1972, Ann. Rev. Astron. Astrophys., 10, 101.

Fricke, W.: 1985, Celest. Mech., 36, 207.

Guinot, B. et al.: 1961, Bull. Astron., 23, 307.

IAU Commission 8: 1986, Transactions of the IAU, XIXB, 111.

Li Qi: 1987, Astron. Astrophys., 174, 307. 